\title{
Секвенциальные аксиоматизации простых паралогик ${ }^{1}$
}

\author{
В. м. Попов
}

\author{
Памяти российского философа и логика \\ Владимира Александровича Смирнова, \\ который внес неоценимый вклад в исследования \\ секвенииальных аксиоматизаций логических систем, \\ посвящается
}

\begin{abstract}
The sequent systems axiomatizing some simple paralogics are presented and the solution of the decision problem for these simple paralogics is given. The connection of these logics with the classical propositional logic and the intuitionistic propositional logic is described.

Ключевые слова: секвенция, исчисление, пересечение логик, паралогика
\end{abstract}

Формулируются удобные для поиска доказательства секвенциальные исчисления, аксиоматизирующие простые паралогики. Положительно решается вопрос о разрешимости паралогик, аксиоматизируемых посредством этих секвенциальных исчислений. Устанавливается связь между рассматриваемыми простыми паралогиками с одной стороны и классической и интуиционистской пропозициональными логиками с другой стороны.

Язык $L$, являющийся языком всех рассматриваемых в предлагаемой работе логик, есть стандартно определяемый пропозициональный язык, алфавиту которого принадлежат только следующие символы: $p_{1}, p_{2}, \ldots$ (пропозициональные переменные языка $L), \&, \vee, \supset$ (бинарные логические связки языка $L), \neg$ (унарная логическая связка языка $L$ ), левая и правая круглые скобки. Определение $L$-формулы индуктивно: (1) всякая пропозициональная переменная языка $L$ есть $L$-формула, (2) если $A$

\footnotetext{
${ }^{1}$ Работа выполнена при поддержке РГНФ, проект № 06-03-00020а.
} 
и $B$ являются $L$-формулами, то $(A \& B),(A \vee B),(A \supset B)$ и $(\neg A)$ являются $L$-формулами, (3) ничто другое не является $L$ формулой. Принимаем обычные соглашения об опускании скобок в $L$-формулах и используем «формула» как сокращение для «L-формула». Квазиэлементарной формулой называем формулу, в которую не входит ни одна бинарная логическая связка языка $L$. Длиной квазиэлементарной формулы называем число всех вхождений $\neg$ в эту формулу. Ясно, что для всякой квазиэлементарной формулы существует единственная длина этой квазиэлементарной формулы, и что длина всякой квазиэлементарной формулы есть целое неотрицательное число. Обозначаем правило модус поненс в $L$ через $M P$, а правило подстановки формулы в формулу вместо пропозициональной переменной языка $L$ обозначаем через $S u b$. Логикой называем непустое множество формул, замкнутое относительно $M P$ и $S u b$. Теорией логики $L$ называем множество формул, включающее логику $L$ и замкнутое относительно $M P$. Понятно, что множество всех формул является логикой, а также теорией любой логики. Для всякой логики $L$ называем множество всех формул тривиальной теорией логики $L$. Противоречивой теорией логики $L$ называем такую теорию $T$ логики $L$, что для некоторой формулы $A$ верно: $A \in T$ и $\neg A \in T$. Паранепротиворечивой теорий логики $L$ называем такую противоречивую теорию $T$ логики $L$, что $T$ не есть тривиальная теория логики $L$. Паранепротиворечивой логикой называем такую логику $L$, что существует паранепротиворечивая теория логики $L$. Простой паранепротиворечивой логикой называем такую паранепротиворечивую логику $L$, что для всякой паранепротиворечивой теории $T$ логики $L$ верно: если $A \in T$ и $\neg A \in T$, то $A$ есть квазиэлементарная формула. Полной теорией логики $L$ называем такую теорию $T$ логики $L$, что для всякой формулы $A$ верно следующее: $A \in T$ или $\neg A \in T$. Параполной теорией логики $L$ называем такую теорию $T$ логики $L$, что $T$ не является полной теорией логики $L$ и всякая полная теория логики $L$, включающая $T$, есть тривиальная теория логики $L$. Параполной логикой называем такую логику $L$, что существует параполная теория логики $L$. Простой параполной логикой называем такую параполную логику $L$, что для всякой параполной теории $T$ логики $L$ верно следующее: существует такая квази- 
элементарная формула $A$, что ни $A$ и ни $\neg A$ не принадлежат $T$. Простой паралогикой называем такую логику, которая является простой паранепротиворечивой логикой или простой параполной логикой. Простой паранормальной логикой называем такую логику, которая является простой паранепротиворечивой логикой и простой параполной логикой.

Для всякого $i$ из $\{0,1,2,3\}$ определим исчисления $H \operatorname{Int}_{i, 0}$, $H$ Int $_{i, 1}$, Int $_{i, 2}, \ldots$ Int $_{i, \omega}, H I_{i, 0}, H I_{i, 1}, H I_{i, 2}, \ldots H I_{i, \omega}$. Все эти исчисления являются исчислениями гильбертовского типа, язык каждого из которого есть $L$, каждое из этих исчислений имеет единственное правило вывода - правило $M P$. Во всяком из этих исчислений выводы (в частности, доказательства) строятся обычным для гильбертовского типа исчислений образом. Теперь для определения любого из указанных исчислений остается задать множество всех его аксиом.

Множеству всех аксиом исчисления $\operatorname{HInt}_{0, k}(k$ есть целое неотрицательное число) принадлежат все те и только те формулы, каждая из которых имеет хотя бы один из следующих видов (здесь и далее $A, B, C$ и $D$-формулы):

(I) $(A \supset B) \supset((B \supset C) \supset(A \supset C))$, (II) $A \supset(A \vee B)$, (III) $B \supset(A \vee B),(\mathrm{IV})(A \supset C) \supset((B \supset C) \supset((A \vee B) \supset C)$, $(\mathrm{V})(A \& B) \supset A,(\mathrm{VI})(A \& B) \supset B,(\mathrm{VII})(C \supset A) \supset((C \supset$ $B) \supset(C \supset(A \& B)))$, (VIII) $(A \supset(B \supset C)) \supset((A \& B) \supset C)$, (IX) $((A \& B) \supset C) \supset(A \supset(B \supset C)),(\mathrm{X}, k) \neg E \supset(E \supset A)$, где $E$ есть формула, не являющаяся квазиэлементарной формулой, длина которой меньше $k,(\mathrm{XI}, k)(E \supset \neg(A \supset A)) \supset \neg E$, где $E$ есть формула, не являющаяся квазиэлементарной формулой, длина которой меньше $k$.

Множеству всех аксиом исчисления $\operatorname{HInt}_{3, k}$ ( $k$ есть целое неотрицательное число) принадлежат все те и только те формулы, каждая из которых является аксиомой исчисления $H \operatorname{Int}_{0, k}$ или имеет вид $(\neg A \supset(A \supset B)) \vee((C \supset \neg(D \supset D)) \supset \neg C)$.

Множеству всех аксиом исчисления $\operatorname{HInt}_{1, k}$ ( $k$ есть целое неотрицательное число) принадлежат все те и только те формулы, каждая из которых имеет хотя бы один из видов (I)-(IX), или имеет вид $(\mathrm{X}, k)$, или имеет вид $(B \supset \neg(A \supset A)) \supset \neg B$.

Множеству всех аксиом исчисления $\operatorname{HInt}_{2, k}$ ( $k$ есть целое неотрицательное число) принадлежат все те и только те формулы, 
каждая из которых имеет хотя бы один из видов (I)-(IX), или имеет вид $\neg B \supset(B \supset A)$ или имеет вид $(\mathrm{XI}, k)$.

Множеству всех аксиом исчисления $H \operatorname{Int}_{0, \omega}$ принадлежат все те и только те формулы, каждая из которых имеет хотя бы один из видов (I)-(IX), или имеет вид (XII) $\neg F \supset(F \supset A)$, где $F$ есть формула, не являющаяся квазиэлементарной формулой, или имеет вид (XIII) $(F \supset \neg(A \supset A)) \supset \neg F$, где $F$ есть формула, не являющаяся квазиэлементарной формулой.

Множеству всех аксиом исчисления $\operatorname{Hint}_{3, \omega}$ принадлежат все те и только те формулы, каждая из которых является аксиомой исчисления Int $_{0, \omega}$, или имеет вид $(\neg A \supset(A \supset B)) \vee((C \supset$ $\neg(D \supset D)) \supset \neg C)$.

Множеству всех аксиом исчисления $\operatorname{HInt}_{1, \omega}$ принадлежат все те и только те формулы, каждая из которых имеет хотя бы один из видов (I)-(IX), или имеет вид (XII), или имеет вид $(B \neg(A \supset$ A)) $\supset \neg B$.

Множеству всех аксиом исчисления $\operatorname{HInt}_{2, \omega}$ принадлежат все те и только те формулы, каждая из которых имеет хотя бы один из видов (I)-(IX), или имеет вид $\neg B \supset(B \supset A)$, или имеет вид (XIII).

Для всякого $i$ из $\{0,1,2,3\}$ и для всякого $\alpha$ из $\{0,1,2, \ldots \omega\}$ множество всех аксиом исчисления $H I_{i, \alpha}$ равно объединению множества всех аксиом исчисления $H$ Int $_{i, \alpha}$ с множеством всех формул вида $((A \supset B) \supset A) \supset A$.

Условимся, что для всякого $i$ из $\{0,1,2,3\}$ и для всякого $\alpha$ из $\{0,1,2, \ldots \omega\}$ Int $_{i, \alpha}$ и $I_{i, \alpha}$ являются соответственно множеством всех формул, доказуемых в $H \operatorname{Int}_{i, \alpha}$, и множеством всех формул, доказуемых в $H I_{i, \alpha}$.

Доказаны следующие утверждения 1-17.

УТВЕРЖДЕНИЕ 1. Множество Int 0,0 равно множеству всех интуичионистских тавтологий в языке L.

УТВЕРЖДЕНИЕ 2. Множество $I_{0,0}$ равно множеству всех классических тавтологий в языке $L$.

УТВЕРЖДЕНИЕ 3. Int $_{0,0}=$ Int $_{1,0}=$ Int $_{2,0}=$ Int $_{3,0}$.

УТВЕРЖДЕНИЕ 4. $I_{0,0}=I_{1,0}=I_{2,0}=I_{3,0}$.

УТВЕРЖДЕНИЕ 5 . Множества Int In, $_{0,}$, Int $t_{0,2}, \ldots$ Int $_{0, \omega}$ являются попарно различными простыми паранормальными ло- 
гиками, при этом для всякого $\alpha$ из $\{1,2, \ldots \omega\}$ и для всякого $\beta$ из $\{1,2, \ldots \omega\}$ верно, что если $\alpha$ менъше, чем $\beta$, то Int ${ }_{0, \beta}$ включается в Int $_{0, \alpha}$

УТВЕРЖДЕНИЕ 6. Множества $I_{0,1}, I_{0,2}, \ldots I_{0, \omega}$ являются попарно различными простыми паранормальными логиками, при этом для всякого $\alpha$ из $\{1,2, \ldots \omega\}$ и для всякого $\beta$ из $\{1,2, \ldots \omega\}$ верно, что если $\alpha$ менвше, чем $\beta$, то $I_{0, \beta}$ включаemcя \& $I_{0, \alpha}$.

УТВЕРЖДДЕНИЕ 7. Множества Int ${ }_{3,1}$, Int $_{3,2}, \ldots$ Int $_{3, \omega}$ являются попарно различными простыми паранормальными логиками, при этом для всякого $\alpha$ из $\{1,2, \ldots \omega\}$ и для всякого $\beta$ из $\{1,2, \ldots \omega\}$ верно, что если $\alpha$ менвше, чем $\beta$, то Int $_{3, \beta}$ включается в Int $_{3, \alpha}$.

УТВЕРЖДЕНИЕ 8. Множества $I_{3,1}, I_{3,2}, \ldots I_{3, \omega}$ являются попарно различными простыми паранормальными логиками, при этом для всякого $\alpha$ из $\{1,2, \ldots \omega\}$ и для всякого $\beta$ из $\{1,2, \ldots \omega\}$ верно, что если $\alpha$ менвше, чем $\beta$, то $I_{3, \beta}$ включается в $I_{3, \alpha}$.

УТВЕРЖДЕНИЕ 9. Множества $\left\{\right.$ Int $_{0,1}$, Int $_{0,2}, \ldots$ Int $\left._{0, \omega}\right\}$, $\left\{I_{0,1}, I_{0,2}, \ldots I_{0, \omega}\right\}, \quad\left\{\right.$ Int $_{3,1}$, Int $_{3,2}, \ldots$ Int $\left._{3, \omega}\right\} \quad u\left\{I_{3,1}, I_{3,2}, \ldots I_{3, \omega}\right\}$ попарно несовместимы.

УТВЕРЖДЕНИЕ 10. Множества Int $t_{1,1}$, Int $_{1,2}, \ldots$ Int $_{1, \omega}$ являются попарно различными простыми паранепротиворечивыми логиками, ни одна из которых не является параполной логикой, и при этом для всякого $\alpha$ из $\{1,2, \ldots \omega\}$ и для всякого $\beta$ из $\{1,2, \ldots \omega\}$ верно, что если $\alpha$ менъше, чем $\beta$, то Int I $_{1, \beta}$ включается в Int $_{1, \alpha}$.

УТВЕРЖДЕНИЕ 11. Множества $I_{1,1}, I_{1,2}, \ldots I_{1, \omega}$ являются попарно различными простыми паранепротиворечивыми логиками, ни одна из которых не является параполной логикой, и при этом для всякого $\alpha$ из $\{1,2, \ldots \omega\}$ и для всякого $\beta$ из $\{1,2, \ldots \omega\}$ верно, что если $\alpha$ меньше, чем $\beta$, то $I_{1, \beta}$ включается в $I_{1, \alpha}$.

УТВЕРЖДЕНИЕ 12. Множество $\left\{\right.$ Int $_{1,1}$, Int $_{1,2}, \ldots$ Int $\left._{1, \omega}\right\}$ несовместимо с множеством $\left\{I_{1,1}, I_{1,2}, \ldots I_{1, \omega}\right\}$.

УТВЕРЖДЕНИЕ 13. Множества Int I $_{2,1}$, Int $_{2,2}, \ldots$ Int $_{2, \omega}$ яв- 
ляются попарно различными простыми параполными логиками, ни одна из которых не является паранепротиворечивой логикой, и при этом для всякого $\alpha$ из $\{1,2, \ldots \omega\}$ и для всякого $\beta$ из $\{1,2, \ldots \omega\}$ верно, что если $\alpha$ меньше, чем $\beta$, mо Int $_{2, \beta}$ включается в Int $_{2, \alpha}$.

УТВЕРЖКДЕНИЕ 14. Множества $I_{2,1}, I_{2,2}, \ldots I_{2, \omega}$ являются попарно различными простыми параполными логиками, ни одна из которых не является паранепротиворечивой логикой, и при этом для всякого $\alpha$ из $\{1,2, \ldots \omega\}$ и для всякого $\beta$ из $\{1,2, \ldots \omega\}$ верно, что если $\alpha$ менвше, чем $\beta$, то $I_{2, \beta}$ включается $8 I_{2, \alpha}$.

УТВЕРЖКДЕНИЕ 15. Множество $\left\{\right.$ Int $_{2,1}$, Int $_{2,2}, \ldots$ Int $\left._{2, \omega}\right\}$ несовместимо с множеством $\left\{I_{2,1}, I_{2,2}, \ldots I_{2, \omega}\right\}$.

УТВЕРЖДДНИЕ 16. Для всякого $i$ из $\{0,1,2,3\}$ верно, что Int $_{i, \omega}=\cap\left\{\text { Int }_{i, k}\right\}_{k \in\{1,2, \ldots\}}$ u $I_{i, \omega}=\cap\left\{I_{i, k}\right\}_{k \in\{1,2, \ldots\}}$.

УТВЕРЖДЕНИЕ 17. Для всякого $\alpha$ из $\{1,2, \ldots \omega\}$ верно, что Int $_{1, \alpha} \cap$ Int $_{2, \alpha}=$ Int $_{3, \alpha} u I_{1, \alpha} \cap I_{2, \alpha}=I_{3, \alpha}$.

Определим секвенциальные исчисления, аксиоматизирующие рассматриваемые здесь паралогики. Алфавит А языка всех этих секвенциальных исчислений есть объединение алфавита языка $L$ с двухэлементным множеством $\{,, \rightarrow\}$ символов. Непустой последовательностью формул называем слово в алфавите $\mathbf{A}$, имеющее вид $A_{1}, \ldots, A_{n}$, где $n$ - целое положительное число, а $A_{1}, \ldots, A_{n}$ есть формулы. Заметим, что если $n=1$, то $A_{1}, \ldots, A_{n}$ есть $A_{1}$. Пустой последовательностью формул называем пустое слово. Называем $\pi$ последовательностью формул, если $\pi$ есть пустая последовательность формул или непустая последовательность формул. Секвенцией называем слово в алфавите $\mathbf{A}$, имеющее вид $\pi \rightarrow \rho$, где $\pi$ и $\rho-$ последовательности формул. Для всякого целого положительного числа $n$ называем $n$-посылочным секвенциальным правилом любое подмножество $n+1$-вой декартовой степени множества всех секвенций. Называем $R$ секвенциальным правилом, если для некоторого целого положительного числа $n R$ есть $n$-посылочное секвенциальное правило. Называем П применением секвенциального правила $R$, если $\Pi \in R$. Условимся обозначать через $\Gamma, \Delta, \Sigma$ и $\Theta$ последовательности формул, а через $\Lambda$ - пустую последова- 
тельность формул или формулу. Нам потребуются следующие секвенциальные правила: $R 1, R^{\prime} 1, R^{\prime} 2, R 3, R^{\prime} 3, R 4, R 5, R^{\prime} 5, R 6$, $R^{\prime} 6, R 7, R^{\prime} 7, R 8, R^{\prime} 8, R 9, R^{\prime} 9, R 10, R^{\prime} 10, R 11, R^{\prime} 11, R 12, R^{\prime} 12$, $R 13, R^{\prime} 13, R 14, R^{\prime} 14, R 15, R 15 . k$, где $k$ есть целое положительное число, $R 15 . \omega, R^{\prime} 15, R^{\prime} 15 . k$, где $k$ есть целое положительное число, $R^{\prime} 15 . \omega, R 16, R 16 . k$, где $k$ есть целое положительное число, $R 16 . \omega, R^{\prime} 16, R^{\prime} 16 . k$, где $k$ есть целое положительное число, $R^{\prime} 16 . \omega, R 17, R^{\prime} 17$.

$R 1$ есть секвенциальное правило, каждое применение которого имеет вид $<\Gamma, A, B, \Delta \rightarrow \Theta, \quad \Gamma, B, A, \Delta \rightarrow \Theta>$,

$R^{\prime} 1$ есть секвенциальное правило, каждое применение которого имеет вид $<\Gamma, A, B, \Delta \rightarrow \Lambda, \quad \Gamma, B, A, \Delta \rightarrow \Lambda>$,

$R 2$ есть секвенциальное правило, каждое применение которого имеет вид $<\Gamma \rightarrow \Delta, A, B, \Theta, \Gamma \rightarrow \Delta, B, A, \Theta>$,

$R 3$ есть секвенциальное правило, каждое применение которого имеет вид $<A, A, \Gamma \rightarrow \Theta, A, \Gamma \rightarrow \Theta>$,

$R^{\prime} 3$ есть секвенциальное правило, каждое применение которого имеет вид $<A, A, \Gamma \rightarrow \Lambda, \quad A, \Gamma \rightarrow \Lambda>$,

$R 4$ есть секвенциальное правило, каждое применение которого имеет вид $<\Gamma \rightarrow \Theta, A, A, \quad \Gamma \rightarrow \Theta, A>$,

$R 5$ есть секвенциальное правило, каждое применение которого имеет вид $<\Gamma \rightarrow \Theta, \quad A, \Gamma \rightarrow \Theta>$,

$R^{\prime} 5$ есть секвенциальное правило, каждое применение которого имеет вид $<\Gamma \rightarrow \Lambda, \quad A, \Gamma \rightarrow \Lambda>$,

$R 6$ есть секвенциальное правило, каждое применение которого имеет вид $<\Gamma \rightarrow \Theta, \quad \Gamma \rightarrow \Theta, A>$,

$R^{\prime} 6$ есть секвенциальное правило, каждое применение которого имеет вид $<\Gamma \rightarrow, \quad \Gamma \rightarrow A>$,

$R 7$ есть секвенциальное правило, каждое применение которого имеет вид $<A, \Gamma \rightarrow \Theta, \quad A \& B, \Gamma \rightarrow \Theta>$,

$R^{\prime} 7$ есть секвенциальное правило, каждое применение которого имеет вид $<A, \Gamma \rightarrow \Lambda, \quad A \& B, \Gamma \rightarrow \Lambda>$,

$R 8$ есть секвенциальное правило, каждое применение которого имеет вид $<A, \Gamma \rightarrow \Theta, \quad B \& A, \Gamma \rightarrow \Theta>$,

$R^{\prime} 8$ есть секвенциальное правило, каждое применение которого имеет вид $<A, \Gamma \rightarrow \Lambda, \quad B \& A, \Gamma \rightarrow \Lambda>$,

$R 9$ есть секвенциальное правило, каждое применение которого имеет вид $<\Gamma \rightarrow \Theta, A, \quad \Gamma \rightarrow \Theta, B, \quad \Gamma \rightarrow \Theta, A \& B>$, 
$R^{\prime} 9$ есть секвенциальное правило, каждое применение которого имеет вид $<\Gamma \rightarrow A, \quad \Gamma \rightarrow B, \quad \Gamma \rightarrow A \& B>$,

$R 10$ есть секвенциальное правило, каждое применение которого имеет вид $<A, \Gamma \rightarrow \Theta, B, \Gamma \rightarrow \Theta, B, A \vee B, \Gamma \rightarrow \Theta>$,

$R^{\prime} 10$ есть секвенциальное правило, каждое применение которого имеет вид $<A, \Gamma \rightarrow \Lambda, B, \Gamma \rightarrow \Lambda, B, \quad A \vee B, \Gamma \rightarrow \Lambda>$,

$R 11$ есть секвенциальное правило, каждое применение которого имеет вид $<\Gamma \rightarrow \Theta, A, \quad \Gamma \rightarrow \Theta, A \vee B>$,

$R^{\prime} 11$ есть секвенциальное правило, каждое применение которого имеет вид $<\Gamma \rightarrow A, \quad \Gamma \rightarrow A \vee B>$,

$R 12$ есть секвенциальное правило, каждое применение которого имеет вид $<\Gamma \rightarrow \Theta, A, \quad \Gamma \rightarrow \Theta, B \vee A>$,

$R^{\prime} 12$ есть секвенциальное правило, каждое применение которого имеет вид $<\Gamma \rightarrow A, \quad \Gamma \rightarrow B \vee A>$,

$R 13$ есть секвенциальное правило, каждое применение которого имеет вид

$$
<\Gamma \rightarrow \Theta, A, B, \Sigma \rightarrow \Delta, A \supset B, \Gamma, \Sigma \rightarrow \Theta, \Delta>,
$$

$R^{\prime} 13$ есть секвенциальное правило, каждое применение которого имеет вид $<\Gamma \rightarrow A, \quad B, \Sigma \rightarrow \Lambda, \quad A \supset B, \Gamma, \Sigma \rightarrow \Lambda>$,

$R 14$ есть секвенциальное правило, каждое применение которого имеет вид $<A, \Gamma \rightarrow \Theta, B, \quad \Gamma \rightarrow \Theta, A \supset B>$,

$R^{\prime} 14$ есть секвенциальное правило, каждое применение которого имеет вид $<A, \Gamma \rightarrow B, \quad \Gamma \rightarrow A \supset B>$,

$R 15$ есть секвенциальное правило, каждое применение которого имеет вид $<\Gamma \rightarrow \Theta, A, \neg A, \Gamma \rightarrow \Theta>$.

Для всякого целого положительного числа $k R 15 . k$ есть секвенциальное правило, каждое применение которого имеет вид $<\Gamma \rightarrow \Theta, E, \neg E, \Gamma \rightarrow \Theta>$, где $E$ есть формула, не являющаяся квазиэлементарной формулой, длина которой меньше $k$.

$R 15 . \omega$ есть секвенциальное правило, каждое применение которого имеет вид $<\Gamma \rightarrow \Theta, F, \neg F, \Gamma \rightarrow \Theta>$, где $F$ есть формула, не являющаяся квазиэлементарной формулой.

$R^{\prime} 15$ есть секвенциальное правило, каждое применение которого имеет вид $<\Gamma \rightarrow A, \neg A, \Gamma \rightarrow>$.

Для всякого целого положительного числа $k R^{\prime} 15 . k$ есть секвенциальное правило, каждое применение которого имеет вид $<\Gamma \rightarrow E, \neg E, \Gamma \rightarrow>$, где $E$ есть формула, не являющаяся квазиэлементарной формулой, длина которой меньше $k$. 
$R^{\prime} 15 \omega$ есть секвенциальное правило, каждое применение которого имеет вид $<\Gamma \rightarrow F, \neg F, \Gamma \rightarrow>$, где $F$ есть формула, не являющаяся квазиэлементарной формулой.

$R 16$ есть секвенциальное правило, каждое применение которого имеет вид $<A, \Gamma \rightarrow \Theta, \Gamma \rightarrow \Theta, \neg A>$.

Для всякого целого положительного числа $k R 16 . k$ есть секвенциальное правило, каждое применение которого имеет вид $<E, \Gamma \rightarrow \Theta, \quad \Gamma, \Theta \rightarrow \neg E>$, где $E$ есть формула, не являющаяся квазиэлементарной формулой, длина которой меньше $k$.

$R 16 . \omega$ есть секвенциальное правило, каждое применение которого имеет вид $<F, \Gamma \rightarrow \Theta, \Gamma \rightarrow \Theta, \neg F>$, где $F$ есть формула, не являющаяся квазиэлементарной формулой.

$R^{\prime} 16$ есть секвенциальное правило, каждое применение которого имеет вид $<A, \Gamma \rightarrow, \quad \Gamma \rightarrow \neg A>$.

Для всякого целого положительного числа $k R^{\prime} 16 . k$ есть секвенциальное правило, каждое применение которого имеет вид $<E, \Gamma \rightarrow, \Gamma \rightarrow \neg E>$, где $E$ есть формула, не являющаяся квазиэлементарной формулой, длина которой меньше $k$.

$R^{\prime} 16 \omega$ есть секвенциальное правило, каждое применение которого имеет вид $<F, \Gamma \rightarrow, \quad \Gamma \rightarrow \neg F>$, где $F$ есть формула, не являющаяся квазиэлементарной формулой.

$R 17$ есть секвенциальное правило, каждое применение которого имеет вид $<\Gamma \rightarrow \Delta, A, \quad A, \Sigma \rightarrow \Theta, \quad \Gamma, \Sigma \rightarrow \Delta, \Theta>$.

$R^{\prime} 17$ есть секвенциальное правило, каждое применение которого имеет вид $<\Gamma \rightarrow A, \quad A, \Sigma \rightarrow \Lambda, \Gamma, \Sigma \rightarrow \Lambda>$.

Поскольку во всяком из определяемых ниже секвенциальных исчислений доказательства строятся обычным для этого типа исчислений образом (см. [2]) и для всякого формулируемого в предлагаемой работе секвенциального исчисления определение доказуемой в нем секвенции стандартно (см. [2]), то для задания любого из этих исчислений достаточно указать множество всех его основных секвенций и множество всех его правил.

Секвенциальное исчисление $\operatorname{GInt}_{0, k}$, где $k$ - целое положительное число.

Множество всех основных секвенций исчисления GInt $_{0, k}$ есть множество всех секвенций, каждая из которых имеет вид $A \rightarrow$ $A$. Множество $\mathbf{R}_{\mathbf{k}}^{\prime}$ всех правил этого исчисления есть $\left\{R^{\prime} 1, R^{\prime} 3\right.$, $R^{\prime} 5, R^{\prime} 6, R^{\prime} 7, R^{\prime} 8, R^{\prime} 9, R^{\prime} 10, R^{\prime} 11, R^{\prime} 12, R^{\prime} 13, R^{\prime} 14, R 15 . k$, 
$\left.R^{\prime} 16 . k, R^{\prime} 17\right\}$. Доказано следующее утверждение 18.

УТВЕРЖДЕНИЕ 18. Для всякого целого положительного числа $k$ и для всякой формулы $A$ верно: в GInt $_{0, k}$ доказуема секвениия $\rightarrow A$ тогда и только тогда, когда $A \in$ Int $_{0, k}$.

Секвенциальные исчисления $\operatorname{GInt}_{3, k}$, где $k$ - целое положительное число.

Множество всех основных секвенций исчисления $\operatorname{Int}_{3, k}$ есть множество всех секвенций, каждая из которых имеет вид $A \rightarrow A$ или имеет вид $\rightarrow(((\neg A) \supset(A \supset B)) \vee((C \supset(\neg(D \supset D))) \supset$ $(\neg C))$ ). Множество всех правил этого исчисления есть $\mathbf{R}_{\mathbf{k}}^{\prime}$. Доказано следующее утверждение 19.

УТВЕРЖДЕНИЕ 19. Для всякого целого положительного числа $k$ и для всякой формулы $А$ верно: в GInt $_{3, k}$ доказуема секвенчия $\rightarrow A$ тогда и только тогда, когда $A \in$ Int $_{3, k}$.

Секвенциальное исчисление $\operatorname{GInt}_{1, k}$, где $k$ - целое положительное число.

Множество всех основных секвенций исчисления GInt $_{1, k}$ есть множество всех секвенций, каждая из которых имеет вид $A \rightarrow A$. Множество всех правил этого исчисления есть $\left(\mathbf{R}_{\mathbf{k}}^{\prime} \backslash\right.$ $\left.\left\{R^{\prime} 16 . k\right\}\right) \cup\left\{R^{\prime} 16\right\}$. Доказано следующее утверждение 20 .

УТВЕРЖДЕНИЕ 20. Для всякого целого положительного числа $k$ и для всякой формулы $A$ верно: в GInt $_{1, k}$ доказуема секвениия $\rightarrow$ A тогда и только тогда, когда $A \in$ Int $_{1, k}$.

Секвенциальное исчисление Int $_{2, k}$, где $k$ - целое положительное число.

Множество всех основных секвенций исчисления GInt $_{2, k}$ есть множество всех секвенций, каждая из которых имеет вид $A \rightarrow A$. Множество всех правил этого исчисления есть $\left(\mathbf{R}_{\mathbf{k}}^{\prime} \backslash\right.$ $\left.\left\{R^{\prime} 15 . k\right\}\right) \cup\left\{R^{\prime} 15\right\}$. Доказано следующее утверждение 21 .

УТВЕРЖДЕНИЕ 21. Для всякого целого положительного числа $k$ и для всякой формуль $A$ верно: в GInt $_{2, k}$ доказуема секвениия $\rightarrow$ A тогда и только тогда, когда $A \in$ Int $_{2, k}$.

Секвенциальное исчисление $\operatorname{GInt}_{0, \omega}$.

Множество всех основных секвенций исчисления GInt $_{0, \omega}$ есть множество всех секвенций, каждая из которых имеет вид $A \rightarrow A$. Множество всех правил этого исчисления есть $\left(\mathbf{R}_{\mathbf{k}}^{\prime} \backslash\right.$ 
$\left.\left\{R^{\prime} 15 . k, R^{\prime} 16 . k\right\}\right) \cup\left\{R^{\prime} 15 . \omega, R^{\prime} 16 . \omega\right\}$. Доказано следующее утверждение 22.

УТВЕРЖДЕНИЕ 22. Для всякой формулъ А верно: в GInt доказуема секвениия $\rightarrow A$ тогда и только тогда, когда $A \in$ Int $_{0, \omega}$.

Секвенциальное исчисление $\operatorname{GInt}_{3, \omega}$.

Множество всех основных секвенций исчисления GInt $t_{3, \omega}$ есть множество всех секвенций, каждая из которых имеет вид $A \rightarrow A$ или имеет вид $\rightarrow(((\neg A) \supset(A \supset B)) \vee((C \supset(\neg(D \supset$ $D))) \supset(\neg C)))$. Множество всех правил этого исчисления есть множество всех правил исчисления $\operatorname{GInt}_{0, \omega}$. Доказано следующее утверждение 23.

УТВЕРЖДЕНИЕ 23. Для всякой формуль А верно: в GInt $_{3, \omega}$ доказуема секвениия $\rightarrow A$ тогда и только тогда, когда $A \in$ Int $_{3, \omega}$.

Секвенциальное исчисление $\operatorname{GInt}_{1, \omega}$.

Множество всех основных секвенций исчисления $\operatorname{GInt}_{1, \omega}$ есть множество всех секвенций, каждая из которых имеет вид вид $A \rightarrow A$. Множество всех правил этого исчисления есть $\left(\mathbf{R}_{\mathbf{k}}^{\prime} \backslash\right.$ $\left.\left\{R^{\prime} 15 . k, R^{\prime} 16 . k\right\}\right) \cup\left\{R^{\prime} 15 . \omega, R^{\prime} 16\right\}$. Доказано следующее утверждение 24.

УТВЕРЖКДЕНИЕ 24. Для всякой формуль А верно: в GInt доказуема секвениия $\rightarrow A$ тогда и только тогда, когда $A \in$ Int $_{1, \omega}$.

Секвенциальное исчисление $\operatorname{GInt}_{2, \omega}$.

Множество всех основных секвенций исчисления GInt $_{2, \omega}$ есть множество всех секвенций, каждая из которых имеет вид $A \rightarrow A$. Множество всех правил этого исчисления есть $\left(\mathbf{R}_{\mathbf{k}}^{\prime} \backslash\right.$ $\left.\left\{R^{\prime} 15 . k, R^{\prime} 16 . k\right\}\right) \cup\left\{R^{\prime} 15, R^{\prime} 16 . \omega\right\}$. Доказано следующее утверждение 25.

УТВЕРЖКДЕНИЕ 25. Для всякой формулы А верно: в GInt Д., $_{2}$ доказуема секвениия $\rightarrow A$ тогда и только тогда, когда $A \in$ Int $_{2, \omega}$.

Секвенциальное исчисление $G I_{0, k}$, где $k$ - целое положительное число.

Множество всех основных секвенций исчисления $G I_{0, k}$ есть 
множество всех секвенций каждая из которых имеет вид $A \rightarrow A$. Множество $\mathbf{R}_{\mathbf{k}}$ всех правил этого исчисления есть $\{R 1, R 2, R 3$, R4, R5, R6, R7, R8, R9, R10, R11, R12, R13, R14, R15.k, R16.k, $R 17\}$. Доказано следующее утверждение 26.

УТВЕРЖДЕНИЕ 26. Для всякого целого положительного числа $k$ и для всякой формуль $A$ верно: в $G I_{0, k}$ доказуема секвениия $\rightarrow A$ тогда и только тогда, когда $A \in I_{0, k}$.

Секвенциальное исчисление $G I_{3, k}$, где $k$ - целое положительное число.

Множество всех основных секвенций исчисления $G I_{3, k}$ есть множество всех секвенций каждая из которых имеет вид $A \rightarrow A$ или имеет вид $A, \neg A \rightarrow B, \neg B$. Множество всех правил этого исчисления есть $\mathbf{R}_{\mathbf{k}}$. Доказано следующее утверждение 27 .

УТВЕРЖДЕНИЕ 27. Для всякого целого положительного числа $k$ и для всякой формуль $A$ верно: в $G I_{3, k}$ доказуема секвениия $\rightarrow A$ тогда и только тогда, когда $A \in I_{3, k}$.

Секвенциальное исчисление $G I_{1, k}$, где $k$ - целое положительное число.

Множество всех основных секвенций исчисления $G I_{1, k}$ есть множество всех секвенций каждая из которых имеет вид $A \rightarrow A$. Множество всех правил этого исчисления есть $\left(\mathbf{R}_{\mathbf{k}} \backslash\{R 16 . k\}\right) \cup$ $\{R 16\}$. Доказано следующее утверждение 28.

УТВЕРЖДЕНИЕ 28. Для всякого целого положительного числа $k$ и для всякой формуль $А$ верно: в $G I_{1, k}$ доказуема секвениия $\rightarrow A$ тогда и толъко тогда, когда $A \in I_{1, k}$.

Секвенциальное исчисление $G I_{2, k}$, где $k$ - целое положительное число.

Множество всех основных секвенций исчисления $G I_{2, k}$ есть множество всех секвенций каждая из которых имеет вид $A \rightarrow A$. Множество всех правил этого исчисления есть $\left(\mathbf{R}_{\mathbf{k}} \backslash\{R 15 . k\}\right) \cup$ $\{R 15\}$. Доказано следующее утверждение 29.

УТВЕРЖДЕНИЕ 29. Для всякого целого положительного числа $k$ и для всякой формуль $А$ верно: в $G I_{2, k}$ доказуема секвениия $\rightarrow A$ тогда и только тогда, когда $A \in I_{2, k}$.

Секвенциальное исчисление $G I_{0, \omega}$.

Множество всех основных секвенций исчисления $G I_{0, \omega}$ есть 
множество всех секвенций, каждая из которых имеет вид $A \rightarrow$ $A$. Множество всех правил этого исчисления есть $\{R 1, R 2, R 3$,

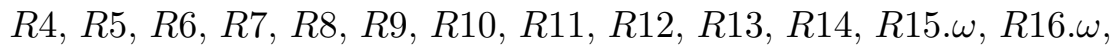
$R 17\}$. Доказано следующее утверждение 30.

УТВЕРЖДЕНИЕ 30. Для всякой формулы $А$ верно: в $G I_{0, \omega}$ доказуема секвениия $\rightarrow A$ тогда и только тогда, когда $A \in I_{0, \omega}$.

Секвенциальное исчисление $G I_{3, \omega}$.

Множество всех основных секвенций исчисления $G I_{3, \omega}$ есть множество всех секвенций, каждая из которых имеет вид $A \rightarrow A$ или имеет вид $A, \neg A \rightarrow B, \neg B$. Множество всех правил этого исчисления есть множество всех правил исчисления $G I_{0, \omega}$. Доказано следующее утверждение 31.

УТВЕРЖДЕНИЕ 31. Для всякой формуль А верно: в $G I_{3, \omega}$ доказуема секвениия $\rightarrow A$ тогда и только тогда, когда $A \in I_{3, \omega}$.

Секвенциальное исчисление $G I_{1, \omega}$.

Множество всех основных секвенций исчисления $G I_{1, \omega}$ есть множество всех секвенций, каждая из которых имеет вид $A \rightarrow A$. Множество всех правил этого исчисления есть $\left(\mathbf{R}_{\mathbf{k}} \backslash\right.$ $\{R 15 . k, R 16 . k\}) \cup\{R 15 . \omega, R 16\}$. Доказано следующее утверждение 32 .

УТВЕРЖДЕНИЕ 32.

Для всякой формуль $А$ верно: в $G I_{1, \omega}$ доказуема секвениия $\rightarrow A$ тогда и только тогда, когда $A \in I_{1, \omega}$.

Секвенциальное исчисление $G I_{2, \omega}$.

Множество всех основных секвенций исчисления $G I_{2, \omega}$ есть множество всех секвенций, каждая из которых имеет вид $A \rightarrow A$. Множество всех правил этого исчисления есть $\left(\mathbf{R}_{\mathbf{k}} \backslash\right.$ $\{R 15 . k, R 16 . k\}) \cup\{R 15, R 16 . \omega\}$. Доказано следующее утверждение 33 .

УТВЕРЖДЕНИЕ 33. Для всякой формулъ А верно: в $G I_{2, \omega}$ доказуема секвениия $\rightarrow A$ тогда и только тогда, когда $A \in I_{2, \omega}$.

Обозначаем через $G$ объединение множества $\left\{\right.$ Int $\left._{i, \alpha}\right\}(i \in$ $\{0,1,2\}$ и $\alpha \in\{1,2,3, \ldots \omega\})$ с множеством $\left\{I_{j, \beta}\right\}(j \in\{0,1,2,3\}$ и $\beta \in\{1,2,3, \ldots \omega\})$.

Для каждого $V$ из $G$ определяем напарника исчисления $V$ как такое секвенциальное исчисление $W$, что (1) язык исчисле- 
ния $W$ есть язык исчисления $V,(2)$ множество всех основных секвенций исчисления $W$ есть множество всех основных секвенций исчисления $V,(3)$ множество всех правил исчисления $W$ есть разность множества всех правил исчисления $V$ с множеством $\left\{R 17, R^{\prime} 17\right\},(4)$ доказательства в $W$ строятся обычным для секвенциального типа исчислений образом. Ясно, что для всякого $V$ из $G$ существует единственный напарник исчисления $V$. Для всякого $V$ из $G$ обозначаем через $F C V$ напарника исчисления $V$. Следующее утверждение 34 (теорема об устранимости сечения для исчислений из $G$ ) доказано методом, предложенным и примененным Г. Генценом в [1].

УТВЕРЖДДНИЕ 34. Для всякого $V$ из $G$ и всякой секвениии $S$ верно: $S$ доказуема в $V$ тогда и только тогда, когда $S$ доказуема в $F C V$.

УТВЕРЖКДЕНИЕ 35. Для всякого $V$ из $G$ исчисление $F C V$ разрешимо.

Утверждение 35 доказано методом редуцированных секвенций (см. [1]).

С использованием утверждений $17,20,21,24,25$ и 35 доказано следующее утверждение 36.

УТВЕРЖКДЕНИЕ 36. Для всякого $i$ из $\{0,1,2,3\}$ и для всякого $\alpha$ из $\{1,2, \ldots \omega\}$ логика Int $_{i, \alpha}$ и логика $I_{i, \alpha}$ разрешимы.

Связь между изучаемыми простыми паралогиками с одной стороны и классической $I_{0,0}$ и интуиционистской Int $t_{0,0}$ пропозициональными логиками с другой стороны устанавливают нижеследующие утверждения 37 и 38, а также формулируемые ниже теоремы 39-42.

УТВЕРЖДДНИЕ 37. Для всякого $i$ из $\{0,1,2,3\}$ и для всякого а из $\{1,2, \ldots \omega\}$ позитивный фрагмент логики $I_{i, \alpha}$ равен позитивному фрагменту логики $I_{0,0}$.

УТВЕРЖДЕНИЕ 38. Для всякого $i$ из $\{0,1,2,3\}$ и для всякого $\alpha$ из $\{1,2, \ldots \omega\}$ позитивный фрагмент логики Int $_{i, \alpha}$ равен позитивному фрагменту логики Int I,0 $_{\text {. }}$

Утверждения 37 и 38 доказаны с помощью утверждений 17 , $18,20,21,22,24,25,26,28,29,30,32,33$ и полученных в [1] фундаментальных результатов по секвенциальной аксиоматиза- 
ции интуиционистской и классической логик. Доказаны также следующие теоремы 39-42.

TEOPEMA 39. Пусть $i \in\{0,1,2,3\}, \alpha \in\{1,2, \ldots \omega\}$ u $\varphi$ есть отображение множества всех пропозициональных переменных языка L во множество всех формул, удовлетворяющее условияM:

(1) $\varphi(p)$ не есть пропозичиональная переменная языка $L$ ни для какой пропозициональной переменной $p$ языка $L$,

(2) для всякой пропозициональной переменной $p$ языка $L$ $p \supset \varphi(p) u \varphi(p) \supset p$ принадлежат логике Int $_{i, \alpha}$.

Пусть $h_{\varphi}$ есть такое отображение множества всех формул в себл, что для всякой пропозициональной переменной $р$ языка $L$ и для всяких формул $B$ и $C$ выполняются следуюшие условия:

(a) $h_{\varphi}(p)=\varphi(p)$,

(b) $h_{\varphi}((B \circ C))=\left(h_{\varphi}(B) \circ h_{\varphi}(C)\right)$, где $\circ \in\{\&, \vee, \supset\}$,

(c) $h_{\varphi}((\neg B))=\left(\neg h_{\varphi}(B)\right)$.

Тогда для всякой формуль $A$ верно: $A \in$ Int $_{0,0}$ тогда и только тогда, когда $h_{\varphi}(A) \in$ Int $_{i, \alpha}$.

TEOPEMA 40. Пусть $i \in\{0,1,2,3\}, \alpha \in\{1,2, \ldots \omega\}$ u g есть такое отображение множества всех формул в себл, что для всякой пропозичиональной переменной $p$ языка $L$ и для всяких формул В и С выполняется условия:

(a) $g(p)=p$,

(b) $g((B \circ C))=(g(B) \circ g(C))$, где $\circ \in\{\&, \vee, \supset\}$,

(c) $g((\neg B))=\left(g(B) \supset\left(\neg\left(p_{1} \supset p_{1}\right)\right)\right)$.

Тогда для всякой формуль $A$ верно: $A \in$ Int $_{0,0}$ тогда и только тогда, когда $g(A) \in$ Int $_{i, \alpha}$.

TEOPEMA 41. Пусть $i \in\{0,1,2,3\}, \alpha \in\{1,2, \ldots \omega\}$ u $\varphi$ есть отображение множества всех пропозичиональных переменных языка L во множество всех формул, удовлетворяющее условиЯMN: 
(1) $\varphi(p)$ не есть пропозициональная переменная языка $L$ ни для какой пропозищионалъной переменной $р$ языка $L$,

(2) для всякой пропозициональной переменной $p$ языка $L$ $p \supset \varphi(p)$ u $\varphi(p) \supset p$ принадлежат логике $I_{i, \alpha}$.

Пусть $h_{\varphi}$ есть такое отображение множества всех формул в себя, что для всякой пропозициональной переменной $р$ языка $L$ и для всяких формул $B$ и $C$ выполняются следующие условия:

(a) $h_{\varphi}(p)=\varphi(p)$

(b) $h_{\varphi}((B \circ C))=\left(h_{\varphi}(B) \circ h_{\varphi}(C)\right)$, где ० $\in\{\&, \vee, \supset\}$,

(c) $h_{\varphi}((\neg B))=\left(\neg h_{\varphi}(B)\right)$.

Тогда для всякой бормуль $A$ верно: $A \in I_{0,0}$ тогда и только тогда, когда $h_{\varphi}(A) \in I_{i, \alpha}$.

TEOPEMA 42. Пусть $i \in\{0,1,2,3\}, \alpha \in\{1,2, \ldots \omega\}$ u g ecms такое отображение множества всех формул в себя, что для всякой пропозичиональной переменной $p$ языка $L$ и для всяких бормул В и $С$ выполняется условия:

(a) $g(p)=p$,

(b) $g((B \circ C))=(g(B) \circ g(C))$, где $\circ \in\{\&, \vee, \supset\}$,

(c) $g((\neg B))=\left(g(B) \supset\left(\neg\left(p_{1} \supset p_{1}\right)\right)\right)$.

Тогда для всякой формуль $A$ верно: $A \in I_{0,0}$ тогда и толъко тогда, когда $g(A) \in I_{i, \alpha}$.

\section{Литература}

[1] Генцен Г. Исследование логических выводов //Математическая теория логического вывода. М., 1967. С.9-74.

[2] Смирнов В. А. Формальный вывод и логические исчисления// Смирнов В. А. Теория логического вывода. М., 1999. С.16-233. 